\title{
An exponential inequality for associated variables
}

\author{
Paulo Eduardo Oliveira*,1 \\ Dep. Matemática, Univ. Coimbra, Apartado 3008, 3001-454 Coimbra, Portugal \\ Received 2 April 2004; accepted 22 November 2004 \\ Available online 16 February 2005
}

\begin{abstract}
We prove an exponential inequality for positively associated and strictly stationary random variables replacing an uniform boundedness assumption by the existence of Laplace transforms. The proof uses a truncation technique together with a block decomposition of the sums to allow an approximation to independence. We show that for geometrically decreasing covariances our conditions are fulfilled, identifying a convergence rate for the strong law of large numbers.

(C) 2005 Elsevier B.V. All rights reserved.
\end{abstract}

Keywords: Association; Exponential inequality

\section{Introduction}

One of the main tools used for characterizing convergence rates in nonparametric estimation has been convenient versions of Bernstein type exponential inequalities. There exist several versions available in the literature for independent sequences of variables with assumptions of uniform boundedness or some, quite relaxed, control on their (centered or noncentered) moments. If the independent case is classical in the literature the treatment of dependent variables is more recent. The extension to dependent variables was first studied considering $m$-dependence or different mixing conditions. An exponential inequality for strong mixing variables eventually was

\footnotetext{
*Fax.: + 351239832568

${ }^{1}$ The author was partially supported by Centro de Matemática da Universida de Coimbra, Fundação para a Ciência e Tecnologia (FCT) and POCTI.
} 
proved in Carbon (1993) using the same type, as for the treatment of the independent case, of assumptions on the variables, besides the strong mixing: uniformly bounded or some control on the moments. Naturally, these latter extensions included some extra terms on the upper bounds depending on the mixing coefficients. An account of the main results briefly described before may be found in Bosq (1996). In another direction in controlling dependent variables, Azuma (1967) proved a version of exponential inequalities are also available for martingale differences supposing the variables to be uniformly bounded and, more recently, Lesigne and Volný (2001) obtained an extension assuming only the existence of Laplace transforms. Another dependence structure that has attracted the interest of probabilists and statisticians is association, as introduced by Esary et al. (1967). For this dependence structure the idea of asymptotic independence is not so explicitly stated as in mixing structures. For associated random variables Birkel (1988) seems to have been the first author to prove some moment inequalities. An exponential type inequality appeared much latter in Ioannides and Roussas (1998) under the assumption of uniform boundedness and some convenient behaviour on the covariance structure of the variables. The technique used in this latter reference was adapted in Henriques and Oliveira (2002a) to prove almost sure consistency results in nonparametric distribution function estimation, based on associated samples, with description of convergence rates. In another direction, with a somewhat different method, exponential decay rates for nonparametric density estimation, also based on associated samples, were proved in Henriques and Oliveira (2002b). The present article presents an extension of the Ioannides and Roussas's (1998) inequality dropping the boundedness assumption, which is replaced by the existence of Laplace transforms.

The article is organized as follows: Section 2 describes some auxiliary results and introduces the truncated variables used to approximate the original variables, the corresponding tails and the block decomposition of the sums; Section 3 studies the truncated part giving conditions on the truncating sequence to enable the proof of an exponential inequality for these terms; Section 4 treats the tails left aside from the truncation and, finally, Section 5 summarizes the partial results into a final theorem. As indicated, the proof technique consists on a truncation which is then treated using a blocking decomposition of the sums, together with a control on the tails of the distribution, achieved assuming the existence of Laplace transforms.

\section{Definitions, preliminary results and notation}

We say that the variables $X_{1}, X_{2}, \ldots$ are associated if, for every $n \in \mathbb{N}$ and $f, g: \mathbb{R}^{n} \longrightarrow \mathbb{R}$ coordinatewise increasing

$$
\operatorname{Cov}\left(f\left(X_{1}, \ldots, X_{n}\right), g\left(X_{1}, \ldots, X_{n}\right)\right) \geqslant 0
$$

whenever this covariance exists.

For associated variables there exist some general inequalities justifying the use of assumptions on the covariance structure. One of such inequalities, useful in the sequel, appears in Dewan and Prakasarao (1999) and is a generalization of an earlier result by Newman (1980). It states a version for generating functions of Newman's (1984) inequality for characteristic functions. 
Lemma 2.1. Let $W_{1}, \ldots, W_{n}$ be associated random variables bounded by a constant $M$. Then, for every $\theta>0$

$$
\left|\mathbb{E}\left(\mathrm{e}^{\theta \sum_{i=1}^{n} W_{i}}\right)-\prod_{i=1}^{n} \mathbb{E}\left(\mathrm{e}^{\theta W_{i}}\right)\right| \leqslant \theta^{2} \mathrm{e}^{n \theta M} \sum_{1 \leqslant i<j \leqslant n} \operatorname{Cov}\left(W_{i}, W_{j}\right) .
$$

This inequality was used in Dewan and Prakasarao (1999) to prove an exponential convergence rate for the nonparametric estimator of the density, but the method used to control all the terms involved forced the authors to assume a condition that is unattainable for associated variables. In fact, these authors assumed that $1 / n \sum_{i=1}^{n} \operatorname{Cov}\left(X_{1}, X_{i}\right)$ converges to zero exponentially fast. Now, for associated variables, all the covariances are nonnegative so that, at best, the series $\sum_{i=1}^{\infty} \operatorname{Cov}\left(X_{1}, X_{i}\right)$ is convergent with positive limit, and, in such case, $1 / n \sum_{i=1}^{n} \operatorname{Cov}\left(X_{1}, X_{i}\right)$ converges to zero at the rate $n^{-1}$. That is, only a polynomial decrease rate may be satisfied. The inequality stated in Lemma 2.1 was later used in Henriques and Oliveira (2002b) to prove a version of an exponential rate for the kernel estimator for the density, avoiding the difficulties just described.

We quote next a general lemma used to control some of the terms appearing in the course of proof.

Lemma 2.2 (Devroye, 1991). Let $W$ be a centred random variable. If there exist $a, b \in \mathbb{R}$ such that $\mathrm{P}(a \leqslant W \leqslant b)=1$, then, for every $\lambda>0$,

$$
\mathbb{E}\left(\mathrm{e}^{\lambda W}\right) \leqslant \exp \left(\frac{\lambda^{2}(b-a)^{2}}{8}\right) .
$$

Next we introduce the notation that will be used throughout the text. Let $c_{n}, n \geqslant 1$, be a sequence of nonnegative real numbers such that $c_{n} \longrightarrow+\infty$ and, given the random variables $X_{n}, n \geqslant 1$, define, for each $i, n \geqslant 1$,

$$
\begin{aligned}
& X_{1, i, n}=-c_{n} \rrbracket_{\left(-\infty,-c_{n}\right)}\left(X_{i}\right)+X_{i} \rrbracket_{\left[-c_{n}, c_{n}\right]}\left(X_{i}\right)+c_{n} \rrbracket_{\left(c_{n},+\infty\right)}\left(X_{i}\right), \\
& X_{2, i, n}=\left(X_{i}-c_{n}\right) \rrbracket_{\left(c_{n},+\infty\right)}\left(X_{i}\right), \quad X_{3, i, n}=\left(X_{i}+c_{n}\right) \rrbracket_{\left(-\infty,-c_{n}\right)}\left(X_{i}\right),
\end{aligned}
$$

where $\rrbracket_{A}$ represents the characteristic function of the set $A$. For each $n \geqslant 1$ fixed, the variables $X_{1,1, n}, \ldots, X_{1, n, n}$ are uniformly bounded, thus they may be treated using Lemma 2.1. Note that, for each $n \geqslant 1$ fixed, all these variables are monotone transformations of the initial variables $X_{n}$. This implies that an association assumption is preserved by this construction.

The proof of an exponential inequality will use, besides the truncation introduced before, a convenient decomposition of the sums into blocks. This block decomposition is the mean to an approximation to independence technique on the truncated variables. The tails will be treated directly using Laplace transforms.

Consider a sequence of natural numbers $p_{n}$ such that, for each $n \geqslant 1, p_{n}<n / 2$ and define $r_{n}$ as the greatest integer less or equal to $n / 2 p_{n}$. Define then, for $q=1,2,3$, and $j=1, \ldots, 2 r_{n}$

$$
Y_{q, j, n}=\sum_{l=(j-1) p_{n}+1}^{j p_{n}}\left(X_{q, l, n}-\mathbb{E}\left(X_{q, l, n}\right)\right) .
$$


Finally, for each $q=1,2,3$, and $n \geqslant 1$, define

$$
\begin{aligned}
& Z_{q, n, \mathrm{od}}=\sum_{j=1}^{r_{n}} Y_{q, 2 j-1, n}, \quad Z_{q, n, \mathrm{ev}}=\sum_{j=1}^{r_{n}} Y_{q, 2 j, n}, \\
& R_{q, n}=\sum_{l=2 r_{n} p_{n}+1}^{n}\left(X_{q, l, n}-\mathbb{E}\left(X_{q, l, n}\right)\right) .
\end{aligned}
$$

The proof of the main result is now divided into the control of the bounded terms, corresponding to the index $q=1$, and the control of the nonbounded terms that correspond to the indices $q=2$ and 3 .

\section{Controlling the bounded terms}

From definitions (1) and (2) it is obvious that $\left|Y_{1, j, n}\right| \leqslant 2 p_{n} c_{n}, j=1, \ldots, r_{n}$. This enables us to use Lemma 2.2 to control the Laplace transform of these variables. A straightforward application of this lemma produces the following upper bounds.

Lemma 3.1. Let $X_{1}, X_{2}, \ldots$ be random variables. If $Y_{1, j, n}, j=1, \ldots, 2 r_{n}$ are defined by (2) then, for every $\lambda>0$

$$
\begin{aligned}
& \prod_{j=1}^{r_{n}} \mathbb{E}\left(\mathrm{e}^{\lambda Y_{1,2 j-1, n}}\right) \leqslant \exp \left(\lambda^{2} n p_{n} c_{n}^{2}\right), \\
& \prod_{j=1}^{r_{n}} \mathbb{E}\left(\mathrm{e}^{\lambda Y_{1,2 j, n}}\right) \leqslant \exp \left(\lambda^{2} n p_{n} c_{n}^{2}\right) .
\end{aligned}
$$

As it was done in Ioannides and Roussas (1998) and Henriques and Oliveira (2002a,b) we will be interested in controlling the differences between the Laplace transform of a sum of variables and what we would have if the variables were independent. These are the terms appearing in the left side of the inequalities stated in the previous lemma. This control is achieved by summing the odd indexed terms on one side and the even indexed terms on the other side, as was done in Henriques and Oliveira (2002b).

Lemma 3.2. Let $X_{1}, X_{2}, \ldots$ be strictly stationary and associated random variables. On account of definitions (1), (2) and (3), and for every $\lambda>0$

$$
\left|\mathbb{E}\left(\mathrm{e}^{\lambda Z_{1, n, \mathrm{od}}}\right)-\prod_{j=1}^{r_{n}} \mathbb{E}\left(\mathrm{e}^{\lambda Y_{1,2 j-1, n}}\right)\right| \leqslant \frac{\lambda^{2} n}{2} \mathrm{e}^{\lambda n c_{n}} \sum_{j=p_{n}+2}^{\left(2 r_{n}-1\right) p_{n}} \operatorname{Cov}\left(X_{1}, X_{j}\right)
$$

and analogously for the term corresponding to $Z_{1, n, \mathrm{ev}}$. 
Proof. According to (3) and the fact that the variables defined in (1) are associated we have, from a direct application of Lemma 2.1

$$
\left|\mathbb{E}\left(\mathrm{e}^{\lambda Z_{1, n, \mathrm{od}}}\right)-\prod_{j=1}^{r_{n}} \mathbb{E}\left(\mathrm{e}^{\lambda Y_{1,2 j-1, n}}\right)\right| \leqslant \lambda^{2} r_{n} p_{n} \mathrm{e}^{2 \lambda r_{n} p_{n} c_{n}} \sum_{1 \leqslant j<j^{\prime} \leqslant r_{n}} \operatorname{Cov}\left(Y_{1,2 j-1, n}, Y_{1,2 j^{\prime}-1, n}\right) .
$$

As $2 r_{n} p_{n} \leqslant n$ the factors in front of the summation and the exponent are bounded above by the quantities figuring in (4), so we are left with the sum of the covariances to deal with. Using the stationarity of the variables it follows that:

$$
\sum_{1 \leqslant j<j^{\prime} \leqslant r_{n}} \operatorname{Cov}\left(Y_{1,2 j-1, n}, Y_{1,2 j^{\prime}-1, n}\right)=\sum_{j=1}^{r_{n}-1}\left(r_{n}-j\right) \operatorname{Cov}\left(Y_{1,1, n}, Y_{1,2 j-1, n}\right) .
$$

A further invocation of the stationarity implies that

$$
\begin{aligned}
\operatorname{Cov}\left(Y_{1,1, n}, Y_{1,2 j-1, n}\right)= & \sum_{l=0}^{p_{n}-1}\left(p_{n}-l\right) \operatorname{Cov}\left(X_{1,1, n}, X_{1,2 j p_{n}+l+1, n}\right) \\
& +\sum_{l=1}^{p_{n}-1}\left(p_{n}-l\right) \operatorname{Cov}\left(X_{1, l+1, n}, X_{1,2 j p_{n}+1, n}\right) \\
\leqslant & p_{n} \sum_{l=(2 j-1) p_{n}+2}^{(2 j+1) p_{n}} \operatorname{Cov}\left(X_{1,1, n}, X_{1, l, n}\right) .
\end{aligned}
$$

We now analyze the covariances using the Hoeffding formula (see, for example, Lemma 2 in Lehmann (1966))

$$
\operatorname{Cov}\left(X_{1, i, n}, X_{1, j, n}\right)=\int_{\mathbb{R}^{2}} \mathrm{P}\left(X_{1, i, n}>u, X_{1, j, n}>v\right)-\mathrm{P}\left(X_{1, i, n}>u\right) \mathrm{P}\left(X_{1, j, n}>v\right) \mathrm{d} u \mathrm{~d} v .
$$

According to the truncation made in (1), it easily follows that the integrand function vanishes outside the square $\left[-c_{n}, c_{n}\right]^{2}$. Moreover, for $u, v \in\left[-c_{n}, c_{n}\right]$ we may replace, in the integrand function, the variables $X_{1, i, n}$ and $X_{1, j, n}$ by $X_{i}$ and $X_{j}$, respectively, so that

$$
\begin{aligned}
\operatorname{Cov}\left(X_{1, i, n}, X_{1, j, n}\right) & =\int_{\left[-c_{n}, c_{n}\right]^{2}} \mathrm{P}\left(X_{i}>u, X_{j}>v\right)-\mathrm{P}\left(X_{i}>u\right) \mathrm{P}\left(X_{j}>v\right) \mathrm{d} u \mathrm{~d} v \\
& \leqslant \int_{\mathbb{R}^{2}} \mathrm{P}\left(X_{i}>u, X_{j}>v\right)-\mathrm{P}\left(X_{i}>u\right) \mathrm{P}\left(X_{j}>v\right) \mathrm{d} u \mathrm{~d} v=\operatorname{Cov}\left(X_{i}, X_{j}\right)
\end{aligned}
$$

due to the nonnegativity of the latter integrand function, as follows from the association of the original variables. Inserting this into the inequalities stated earlier, (5) and (6), the lemma follows.

We may now prove an exponential inequality for the sum of odd indexed or even indexed terms. 
Lemma 3.3. Let $X_{1}, X_{2}, \ldots$ be strictly stationary and associated random variables. Suppose that

$$
\frac{n}{p_{n}^{2} c_{n}^{4}} \exp \left(\frac{n}{p_{n} c_{n}}\right) \sum_{j=p_{n}+2}^{\infty} \operatorname{Cov}\left(X_{1}, X_{j}\right) \leqslant C_{0}<\infty
$$

Then, for every $\varepsilon \in(0,1)$,

$$
\mathrm{P}\left(\frac{1}{n}\left|Z_{1, n, \text { od }}\right|>\frac{\varepsilon}{9}\right) \leqslant\left(1+36 C_{0}\right) \exp \left(-\frac{n \varepsilon^{2}}{162 p_{n} c_{n}^{2}}\right)
$$

and analogously for $Z_{1, n, \mathrm{ev}}$.

Proof. Applying Markov's inequality and using the previous lemma we find that, for every $\lambda>0$,

$$
\mathrm{P}\left(\frac{1}{n}\left|Z_{1, n, \text { od }}\right|>\frac{\varepsilon}{9}\right) \leqslant \frac{\lambda^{2} n}{2} \exp \left(\lambda n c_{n}-\lambda n \frac{\varepsilon}{9}\right) \sum_{j=p_{n}+2}^{\left(2 r_{n}-1\right) p_{n}} \operatorname{Cov}\left(X_{1}, X_{l}\right)+\exp \left(\lambda^{2} n p_{n} c_{n}^{2}-\lambda n \frac{\varepsilon}{9}\right) .
$$

Optimizing the exponent in the last term of this upper bound we find $\lambda=\varepsilon / 18 p_{n} c_{n}^{2}$, so that this exponent becomes equal to $-n \varepsilon^{2} / 162 p_{n} c_{n}^{2}$. Replacing this choice of $\lambda$ into the first term of the upper bound and taking into account (13) it follows that

$$
\begin{aligned}
\mathrm{P}\left(\frac{1}{n}\left|Z_{1, n, \text { od }}\right|>\frac{\varepsilon}{9}\right) & \leqslant 36 C_{0} \exp \left(-\frac{n \varepsilon^{2}}{18 p_{n} c_{n}^{2}}\right)+\exp \left(-\frac{n \varepsilon^{2}}{162 p_{n} c_{n}^{2}}\right) \\
& \leqslant\left(1+36 C_{0}\right) \exp \left(-\frac{n \varepsilon^{2}}{162 p_{n} c_{n}^{2}}\right) .
\end{aligned}
$$

To complete the treatment of the bounded terms it remains to control the sum corresponding to the indices following $2 r_{n} p_{n}$, that is, $R_{1, n}$.

Lemma 3.4. Let $X_{1}, X_{2}, \ldots$ be strictly stationary associated variables and suppose that

$$
\frac{n}{c_{n}} \longrightarrow+\infty
$$

Then, on account of definition (3), for $n$ large enough and every $\varepsilon>0$, we have $\mathrm{P}\left(\left|R_{1, n}\right|>n \varepsilon\right)=0$.

Proof. As $R_{1, n}=\sum_{l=2 r_{n} p_{n}+1}^{n}\left(X_{1, l, n}-\mathbb{E}\left(X_{1, l, n}\right)\right)$ it follows that $\left|R_{1, n}\right| \leqslant 2\left(n-2 r_{n} p_{n}\right) c_{n} \leqslant 2 c_{n}$, according to the construction of the sequences $r_{n}$ and $p_{n}$. Now $\mathrm{P}\left(\left|R_{1, n}\right|>n \varepsilon\right) \leqslant \mathrm{P}\left(2>n \varepsilon / c_{n}\right)$ and, using (11), this is zero for $n$ large enough.

In order to prove the almost sure convergence of $1 / n \sum_{i=1}^{n}\left(X_{1, i, n}-\mathbb{E}\left(X_{1, i, n}\right)\right)$ and identify a convergence rate we will allow $\varepsilon$ in the previous lemmas to depend on $n$ in such a way as to define a convergent series in the upper bound. Assume that, for some $\alpha>0$ (we will need to be more precise on the choice of $\alpha$, but that will become apparent later),

$$
\varepsilon_{n}=9 \sqrt{2}\left(\frac{\alpha p_{n} \log n}{n}\right)^{1 / 2} c_{n}
$$


Tracing back the proof of Lemma 3.3, this choice of $\varepsilon_{n}$ means that the optimizing value of $\lambda$ would now be $\lambda=1 / \sqrt{2} c_{n}\left(\alpha \log n / n p_{n}\right)^{1 / 2}$. Inserting these expressions in (10) and repeating the arguments would lead to the following result.

Lemma 3.5. Let $X_{1}, X_{2}, \ldots$ be strictly stationary and associated random variables. Suppose that for some $\alpha>0$,

$$
\frac{\log n}{p_{n} c_{n}^{2}} \exp \left(\left(\frac{\alpha n \log n}{2 p_{n}}\right)^{1 / 2}\right) \sum_{j=p_{n}+2}^{\infty} \operatorname{Cov}\left(X_{1}, X_{j}\right) \leqslant C_{0}<\infty .
$$

Then, for $\varepsilon_{n}$ as in (12), we have

$$
\mathrm{P}\left(\frac{1}{n}\left|Z_{1, n, \mathrm{od}}\right|>\frac{\varepsilon_{n}}{9}\right) \leqslant\left(1+\frac{4}{\alpha} C_{0}\right) \exp (-\alpha \log n),
$$

and analogously for $Z_{1, n, \mathrm{ev}}$.

As for the term $R_{1, n}$, it is treated exactly as in Lemma 3.4. Repeating the arguments used in that lemma we would be left with the term $\mathrm{P}\left(2>n \varepsilon_{n} / c_{n}\right)$. But $n \varepsilon_{n} / c_{n} \sim\left(n p_{n} \log n\right)^{1 / 2} \longrightarrow+\infty$, so the argument of Lemma 3.4 still applies.

We may now state a theorem summarizing the partial results described in the lemmas of this section.

Theorem 3.6. Let $X_{1}, X_{2}, \ldots$ be strictly stationary and associated variables satisfying (13) for some $\alpha>0$. On account of definitions (1), (2) and (3) it follows that, for every $\varepsilon_{n}$ as in (12) and $n$ large enough,

$$
\mathrm{P}\left(\frac{1}{n}\left|\sum_{i=1}^{n}\left(X_{1, i, n}-\mathbb{E}\left(X_{1, i, n}\right)\right)\right|>\frac{\varepsilon_{n}}{3}\right) \leqslant 2\left(1+\frac{4}{\alpha} C_{0}\right) \exp (-\alpha \log n) .
$$

Proof. It suffices to write

$$
\mathrm{P}\left(\frac{1}{n}\left|\sum_{i=1}^{n}\left(X_{1, i, n}-\mathbb{E}\left(X_{1, i, n}\right)\right)\right|>\varepsilon\right) \leqslant \mathrm{P}\left(\frac{1}{n}\left|Z_{1, n, \mathrm{od}}\right|>\frac{\varepsilon}{3}\right)+\mathrm{P}\left(\frac{1}{n}\left|Z_{1, n, \mathrm{ev}}\right|>\frac{\varepsilon}{3}\right)+\mathrm{P}\left(\left|R_{1, n}\right|>\frac{n \varepsilon}{3}\right)
$$

and apply the previous lemmas.

Note that the result just proved implies the convergence to zero of the upper bound in (15) but implies the almost sure convergence to zero of $1 / n \sum_{i=1}^{n}\left(X_{1, i, n}-\mathbb{E}\left(X_{1, i, n}\right)\right)$ only if we may choose $\alpha>1$.

\section{Controlling the unbounded terms}

The variables $X_{2, i, n}$ and $X_{3, i, n}$ are associated but not bounded, even for fixed $n$. This means that Lemma 2.1 may not be applied to the sum of such terms. But we may note that these variables depend only on the tails of the distribution of the original variables. So, by controlling the decrease rate of these tails we may prove an exponential inequality for sums of $X_{2, i, n}$ or $X_{3, i, n}$. For 
this control we will not make use of the block decomposition of the sums $\sum_{i=1}^{n}\left(X_{q, i, n}-\mathbb{E}\left(X_{q, i, n}\right)\right)$ as the condition derived would be exactly the same as the one obtained with a direct treatment (the upper bound derived would be the same, up to the multiplication by a constant).

We have, for $q=2,3$, recalling that the variables are identically distributed

$$
\mathrm{P}\left(\mid \sum_{i=1}^{n}\left(X_{q, i, n}-\mathbb{E}\left(X_{q, i, n}\right)\right)>n \varepsilon\right) \leqslant n \mathrm{P}\left(\left|X_{q, 1, n}-\mathbb{E}\left(X_{q, 1, n}\right)\right|>\varepsilon\right) \leqslant \frac{n}{\varepsilon^{2}} \operatorname{Var}\left(X_{q, 1, n}\right) \leqslant \frac{n}{\varepsilon^{2}} \mathbb{E}\left(X_{q, 1, n}^{2}\right) .
$$

Lemma 4.1. Let $X_{1}, X_{2}, \ldots$ be strictly stationary random variables such that there exists $\delta>0$ satisfying $\sup _{|t| \leqslant \delta} \mathbb{E}\left(\mathrm{e}^{t X_{1}}\right) \leqslant M_{\delta}<+\infty$. Then, on account of definition $(1)$, for $t \in(0, \delta]$,

$$
\mathrm{P}\left(\left|\sum_{i=1}^{n}\left(X_{q, i, n}-\mathbb{E}\left(X_{q, i, n}\right)\right)\right|>n \varepsilon\right) \leqslant \frac{2 M_{\delta} n \mathrm{e}^{-t c_{n}}}{t^{2} \varepsilon^{2}}, \quad q=2,3 .
$$

Proof. According to the inequality stated before this lemma it remains to control $\mathbb{E}\left(X_{q, 1, n}^{2}\right)$. Let us fix $q=2$, the other possible choice being treated analogously. We will set $\bar{F}(x)=\mathrm{P}\left(X_{1}>x\right)$. Now, using Markov's inequality it follows that, for $t \in(0, \delta), \bar{F}(x) \leqslant \mathrm{e}^{-t x} \mathbb{E}\left(\mathrm{e}^{t X_{1}}\right) \leqslant M_{\delta} \mathrm{e}^{-t x}$. Writing the mathematical expectation as a Stieltjes integral and integrating by parts we find

$$
\mathbb{E}\left(X_{2,1, n}^{2}\right)=-\int_{\left(c_{n},+\infty\right)}\left(x-c_{n}\right)^{2} \bar{F}(\mathrm{~d} x)=\int_{c_{n}}^{+\infty} 2\left(x-c_{n}\right) \bar{F}(x) \mathrm{d} x \leqslant 2 M_{\delta} \frac{\mathrm{e}^{-t c_{n}}}{t^{2}}
$$

from which the lemma follows.

Note that for this step the association of the variables is irrelevant.

\section{Strong convergence and rates}

This section summarizes the results obtained earlier. In addition, we show that for geometrically decreasing covariances the assumptions made are fulfilled. In this case we also find explicitly the convergence rate that follows.

Theorem 5.1. Let $X_{1}, X_{2}, \ldots$ be strictly stationary and associated random variables satisfying (13) for some $\alpha>0$. Suppose that $\varepsilon_{n}$ is as in (12) and there exists $\delta>\alpha$ satisfying $\sup _{|t| \leqslant \delta} \mathbb{E}\left(\mathrm{e}^{t X_{1}}\right) \leqslant M_{\delta}<+$ $\infty$. Then, on account of definitions (1), (2) and (3), for $n$ large enough,

$$
\mathrm{P}\left(\left|\frac{1}{n} \sum_{i=1}^{n}\left(X_{i}-\mathbb{E}\left(X_{i}\right)\right)\right|>\varepsilon\right) \leqslant\left(2\left(1+\frac{4}{\alpha} C_{0}\right)+\frac{2 M_{\delta} n^{2}}{9 \alpha^{3} p_{n} \log ^{3} n}\right) \exp (-\alpha \log n) .
$$

Proof. Separate the sum in the left of (17) into three terms, apply (15) and (16) with $\varepsilon_{n} / 3$ in place of $\varepsilon$ for the latter. Then choose $t=\alpha$ and $c_{n}=\log n$ in (16), so that the exponents are equal, and recalculate $\varepsilon_{n}$ for this choice of $c_{n}$.

Notice that this result requires some extra assumptions on the choice of $\alpha$ in order to derive the almost sure convergence with rate $\varepsilon_{n}^{-1} \sim n^{1 / 2} / p_{n}^{1 / 2} \log ^{3 / 2} n$. 
Suppose now that $\operatorname{Cov}\left(X_{1}, X_{n}\right)=\rho_{0} \rho^{n}$, for some $\rho_{0}>0$ and $\rho \in(0,1)$. Then (13) may be rewritten as

$$
\rho_{0} \rho^{p_{n}} \leqslant C_{0} \exp \left(-\left(\frac{\alpha n \log n}{2 p_{n}}\right)^{1 / 2}\right) p_{n} \log n
$$

or equivalently, as

$$
p_{n} \log \rho \leqslant-\left(\frac{\alpha n \log n}{2 p_{n}}\right)^{1 / 2}+\log \left(\frac{C_{0} p_{n} \log n}{\rho_{0}}\right) .
$$

This leads to the choice $p_{n} \sim n^{1 / 3} \log ^{1 / 3} n$ and $\alpha>2 \log ^{2} \rho$. Then, the largest order term in the upper bound of (17) behaves like $n^{5 / 3-\alpha} / \log ^{10 / 3} n$, so we should choose $\alpha>\frac{8}{3}$. The convergence rate that follows is of order $n^{1 / 3} / \log ^{5 / 3} n$. This convergence rate is somewhat slower than the rate obtained in Ioannides and Roussas (1998) where a rate $n^{1 / 3} / \log ^{2 / 3} n$ was proved. But, in this latter reference the authors considered uniformly bounded variables so they needed no truncation. Here we used a truncation of the variables using the sequence $\log n$ which is responsible for our slower convergence rate.

If we suppose that the covariances decrease polynomially the inequality just derived is not strong enough to identify a convergence rate. In fact, in this case we would be led to a choice of $p_{n}$ behaving like $n / \log n$ and this would mean that the corresponding $\varepsilon_{n}$ would not converge to zero.

\section{References}

Azuma, K., 1967. Weighted sums of certain dependent random variables. Tôhoku Math. J. 19, 357-367.

Birkel, T., 1988. The invariance principle for associated processes. Stochastic Process. Appl. 27, 57-71.

Bosq, D., 1996. Nonparametric Statistics for Stochastic Processes. Lecture Notes in Statistics, vol. 110, Springer, Berlin.

Carbon, M., 1993. Une nouvelle inégalité de grandes deviations. Applications. Publ. IRMA Lille 32, XI.

Devroye, L., 1991. Exponential inequalities in nonparametric estimation. In: Roussas, G. (Ed.), Nonparametric Functional Estimation and Related Topics. Kluwer Academic Publishers, Dordrecht, pp. 31-44.

Dewan, I., Prakasa Rao, B.L.S., 1999. A general method of density estimation for associated random variables. J. Nonparametric Statist. 10, 405-420.

Esary, J.D., Proschan, F., Walkup, D.W., 1967. Association of random variables, with applications. Ann. Math. Statist. 38, 1466-1474.

Henriques, C., Oliveira, P.E., 2002a. Convergence rates for the estimation of two-dimensional distribution functions under association and estimation of the covariance of the limit empirical process. Pré-Publicações do Departamento de Matemática da Universidade de Coimbra 02-05. Preprint.

Henriques, C., Oliveira, P.E., 2002b. Exponential rates for kernel density estimation under association. Pré-Publicações do Departamento de Matemática da Universidade de Coimbra 02-23. Preprint.

Ioannides, D.A., Roussas, G.G., 1998. Exponential inequality for associated random variables. Statist. Probab. Lett. $42,423-431$.

Lehmann, E.L., 1966. Some concepts of dependence. Ann. Math. Statist. 37, 1137-1153.

Lesigne, E., Volný, D., 2001. Large deviations for martingales. Stochastic Process. Appl. 96, 143-159.

Newman, C., 1980. Normal fluctuation and the FKG inequalities. Comm. Math. Phys. 74, 119-128.

Newman, C., 1984. Asymptotic independence and limit theorems for positively and negatively dependent random variables. In: Tong, Y.L. (Ed.), Inequalities in Statistics and Probability, IMS Lecture Notes-Monograph Series, vol. 5, pp. 127-140. 